\title{
In-House Standardization of Carba NP Test for Carbapenemase Detection in Gram Negative Bacteria
}

\author{
V. Mangayarkarasi*, Stephen Pradeep Moses, S.R. Swarna, \\ K. Kalaiselvi and S. Shaweez Fathima \\ Department of Microbiology, SRM Medical College Hospital and Research Centre, \\ Kattankulathur-603203, Chennai, India \\ *Corresponding author
}

\section{A B S T R A C T}

Keywords

Carba NP test,

Modified Hodge test,

Epsilometer test,

Carbapenem

resistance, Antibiotic

susceptibility testing

Article Info

Accepted:

20 December 2017

Available Online:

10 January 2018
This study was done to evaluate the performance of Carba NP test for the detection of carbapenemase producing Gram negative bacilli. In total, 330 Gram negative bacilli isolates were subjected to Antibiotic Susceptibility Testing, Epsilometer test, Modified Hodge test with meropenem to determine the presence of resistance among carbapenem group of drugs. 44/ 330(13.3\%) showed resistance pattern. The 44 resistant isolates were then subjected to the Carba NP test and incubated at $37^{\circ} \mathrm{C}$ and results were read after 2 hours. According to the principle of Carba NP test there was a color change in all 44 isolates indicating a sensitivity of $100 \%$. Overall, the Carba NP test is a useful tool for rapid detection of carbapenemase activity.

\section{Introduction}

Carbapenem drugs are a group of $\beta$-lactam antibiotics used in the treatment of infections caused by extended spectrum beta-lactamases (ESBL) producing gram negative bacteria (GNB) and several serious bacterial infections like meningitis, nosocomial pneumonia, nosocomial sinusitis and sepsis. The emergence of multidrug resistance is increasing at an alarming rate among a variety of bacterial species, causing both community acquired and nosocomial infections (Taneja et al., 2015). Over the past decade, the emergence of carbapenem resistant Enterobacteriaceae (CRE) has become a formidable threat to public health. The prevalence of CRE, according to some institutions in epidemic area, varies between $24.7 \%$ and 29.8\%. (Xu et al., 2014) Indian single center studies overall report a prevalence of carbapenem resistance in up to $12-15 \%$ of Enterobacteriaceae. Resistance may be associated with hydrolysis of $\beta$ lactam ring, an over expression of $\beta$ lactactamase with no carbapenemase activity or an expression of carbapenemase. (Porwal et al., 2014) Various carbapenemases have been 
reported in Enterobacteriaceae such as the following types: Klebsiella pneumonia carbapenemase (KPC; Ambler class A); Verona integron encoded encodedmetallo $\beta$ lactamase (VIM), imipenemase (IMI), New Delhi metallo $\beta$ lactamase (NMD) (all Ambler class B), and oxacillinase-48 (OXA-48; Ambler class D) (Nordmann et al., 2011) Potential carbapenemase producers are detected by antibiotic susceptibility testing and phenotypic methods such as Modified Hodge test, these tests are not highly sensitive and specific, hence a newer and rapid method of detecting resistance is needed. A simple rapid test (Carba NP) was developed to identify the hydrolysis of the $\beta$ lactam ring of a carbapenem. (Tijet, 2013) This study was done to evaluate the Carba NP test for the detection and screening of carbapenemase producing bacteria in comparison to phenotypic methods such as antibiotic susceptibility testing, epsilometer MIC test and Modified Hodge test.

\section{Materials and Methods}

The study was conducted at a tertiary care hospital in Tamil Nadu, India. Ethical clearance was obtained.

\section{Bacterial isolates}

A total of 330 isolates of Gram negative bacilli was obtained from various clinical samples (e.g. blood, pus, urine, etc.) of hospitalized patients admitted in the hospital for various infections over a period of 1 year from January 2016 to December 2016. Consent forms were obtained from the patients. The isolates were subcultured onto nutrient agar, blood agar and MacConkey agar and subjected to routine biochemical tests for its identification. Of the 330 isolates collected, $148(45 \%)$ were identified as Escherichia coli, 58(18\%) identified as Pseudomonas spp., $54(16 \%)$ identified as Klebsiella pneumoniae, $34(10 \%)$ identified as Acinetobacter spp.,
24(7\%) identified as Citrobacter spp. and 12(4\%) identified as Proteus spp (Fig. 1) and (Fig. 2). Of the various clinical samples urine was the highest collected, followed by sputum and pus (Fig. 3).

\section{Antimicrobial susceptibility}

The isolates were inoculated in peptone water and incubated at $37^{\circ} \mathrm{C}$ for 30 minutes. It was then compared with 0.5 McFarland's standard, from peptone water the isolates were then streaked unto Mueller Hinton agar by lawn streaking method.

The following antibiotics ertapenem $(10 \mu \mathrm{g})$, imipenem $(10 \mu \mathrm{g})$ and meropenem $(10 \mu \mathrm{g})$ from HI-Media Laboratories, BD Diagnostics Pvt Ltd, India were placed incubated for 18-24 hours. The readings were recorded according to Clinical and Laboratory Standards Institute (CLSI) 2016. Of the 330 isolates tested, 44 showed a resistance to one or more carbapenem drugs.

\section{Modified Hodge test}

The 330 isolates were subjected to Modified Hodge Test for detection of carbapenemase according to Center for Disease Control and Prevention (CDC). An overnight culture suspension of Klebsiella pneumonia ATCC BAA-1705 adjusted to 0.5 McFarland standard was inoculated using a sterile cotton swab on the surface of a Mueller Hinton agar (MHA) (HI-MEDIA, Mumbai, India). After drying, $10 \mu \mathrm{g}$ meropenem disk (HI-MEDIA, Mumbai, India) was placed at the center of the plate and the test strain was streaked from the edge of the disk to the periphery of the plate in four different directions. The plate was incubated at $37^{\circ} \mathrm{C}$ for $18-24$ hours. The presence of a 'cloverleaf shaped' zone of inhibition due to carbapenemase production by the test strain was considered as positive. Out of 330 isolates tested 44 showed positive. 


\section{Epsilometer test}

The test strain was inoculated in peptone water for 30 minutes and compared with McFarlands standard 0.5. It was then streaked unto Mueller Hinton agar (MHA) (HIMEDIA, Mumbai, India) with a sterile cotton swab by lawn culture streaking. MIC E strips of meropenem and ertapenem (HI-MEDIA, Mumbai, India) were placed separately and incubated at $37^{\circ} \mathrm{C}$ for 24 hours.

\section{Carba NP test}

Carba NP test was evaluated on those isolates that showed resistance in disk diffusion method to imipenem, meropenem, ertapenem (or) all 3 drugs. One modification was made, Imipenem - Cilastatin instead of Imipenem Monohydrate was used in this study. (Abdel Ghani et al., 2015) One loopful $(10 \mu \mathrm{L})$ of the tested strain directly recovered from the antibiogram plate was resuspended in a Tris$\mathrm{HCl} 20 \mathrm{mmol} / \mathrm{L}$ lysis buffer, vortexed for 1 minute and further incubated at room temperature for 30 minutes. The bacterial suspension was then centrifuged at $10,000 \times g$ at room temperature for 5 minutes. $30 \mu \mathrm{L}$ of the supernatant bacterial suspension was mixed in a 96-well tray with $100 \mu \mathrm{L}$ of a $1-\mathrm{mL}$ solution made of $3 \mathrm{mg}$ of Imipenem Cilastatin, $\mathrm{pH}$ 7.8, phenol red solution, and 0.1 $\mathrm{mmol} / 1 \mathrm{ZnSO}_{4}$. The phenol red solution was prepared by mixing $2 \mathrm{~mL}$ of a phenol red solution $0.5 \% \quad(\mathrm{wt} / \mathrm{vol})$ with $16.6 \mathrm{ml}$ of distilled water. The $\mathrm{pH}$ value was then adjusted to 7.8 by adding drops of $1 \mathrm{~N} \mathrm{NaOH}$.

A mixture of the phenol red solution and the enzymatic suspension being tested was placed in one well of the 96 well microtitre plate, to the next well an uninoculated solution was added and to the next a positive quality control of Klebsiella pneumonia ATCC BAA-1705 was used. The microtitre plate was then incubated at $37^{\circ} \mathrm{C}$ for 2 hours and results read.

\section{Results and Discussion}

Out of 330 Gram negative bacilli isolates obtained, 44 isolates showed resistance in disk diffusion method, epsilometer MIC test and Modified Hodge test. These 44 isolates were then subjected to Carba NP test and all 44 showed a positive reaction indicating presence of carbapenemase enzymes. Our study showed a prevalence of $13.3 \%$ (44/330) carbapenem resistant Enterobacteriaceae which was higher comparing to a study done in Chennai, India (Kumarasamy et al., 2010). There was a similarity in prevalence for carbapenem resistant Enterobacteriaceae when compared to a study done in Mumbai, India. Urine samples showed the highest percentage similar to the same study done (Nair, 2013). The rapid Carba NP test was a much simpler test to be performed in comparison to the phenotypic methods. It is inexpensive, rapid, reproducible, and highly sensitive and specific and thus eliminates the need for using other techniques to identify carbapenemase producers that are time-consuming and less sensitive or specific. The Carba NP test can efficiently indicate the strains to be further tested by PCR or submitted to sequencing for a detailed identification of the carbapenemase genes, the test could be used in low-income countries that are large reservoirs for carbapenemase producers. (Nordmann Patrice et al., 2012) Another study on 244 carbapenemase and non carbapenemase producing Enterobacteriaceae and Pseudomonas aeruginosa isolates showed a sensitivity and specificity of $80 \%$ and $77.3 \%$, respectively by increasing the bacterial extract (Tijet, 2013). In our study there was a sensitivity and specificity of $100 \%$. Mitra et $a l$., reported that the Carba NP test performed poorly in detecting OXA-48 carbapenemases and needs to be further evaluated as OXA48/OXA-181 strains have been found widespread among Indian subcontinent. (Shetty, 2015) 
Fig.1 Distribution of Gram Negative Bacilli $(n=330)$. The chart shows the various organisms isolated from various clinical samples. E. coli was isolated the most

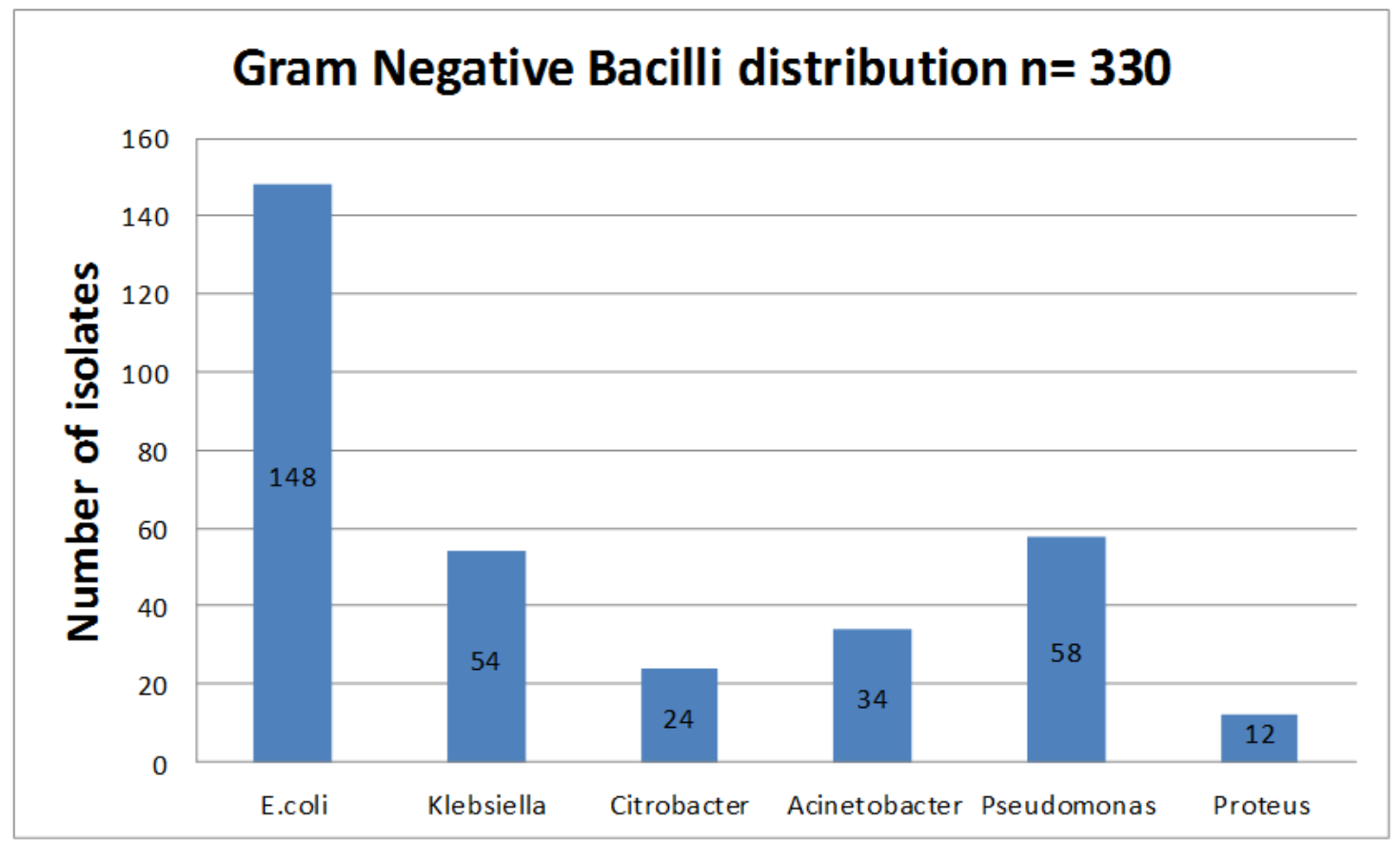

Fig.2 Distribution of various samples collected. Urine samples were collected the most

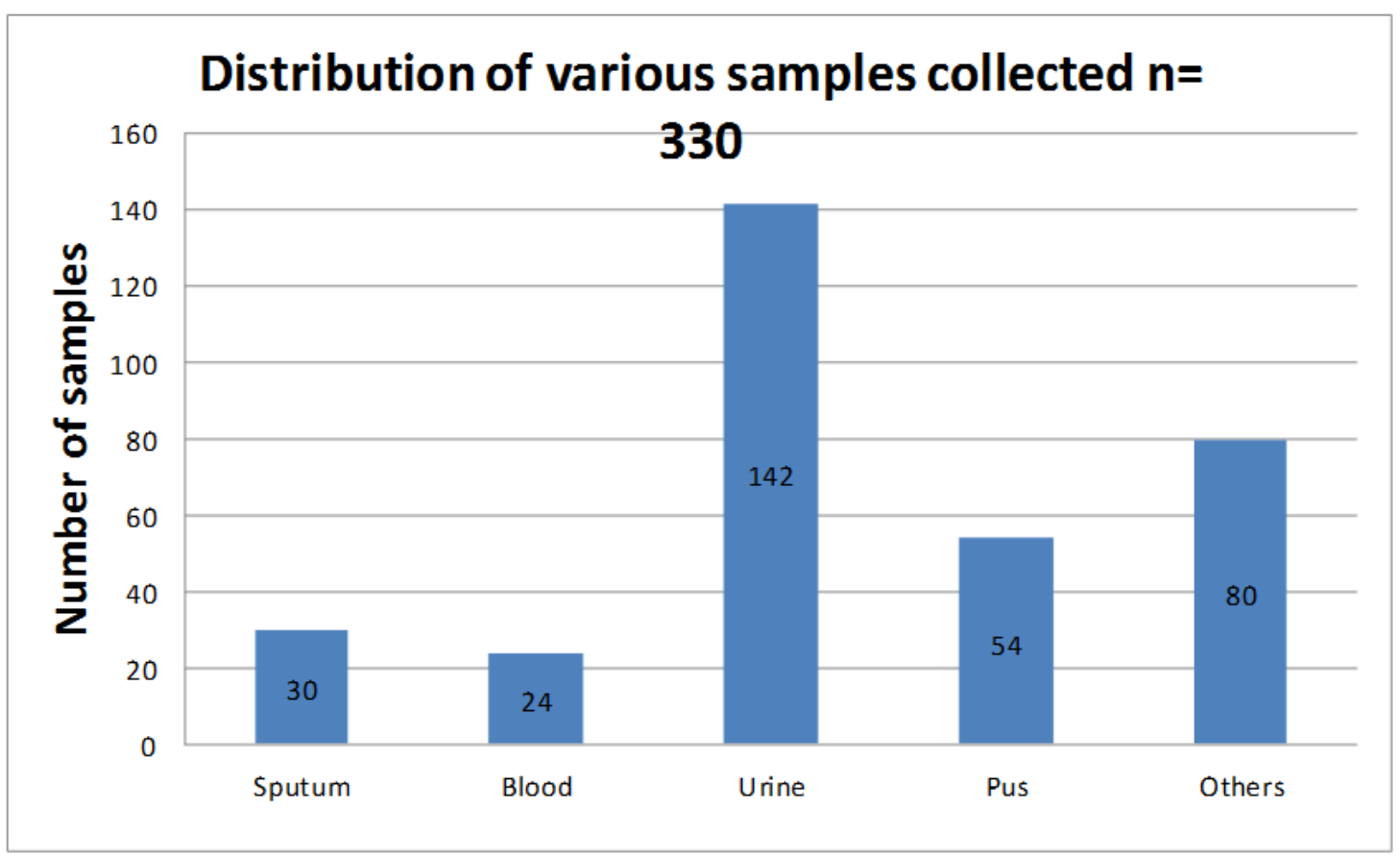


Fig.3 Distribution of carbapenem resistant Gram Negative Bacilli (n=44). $E$. coli isolates showed the maximum resistance

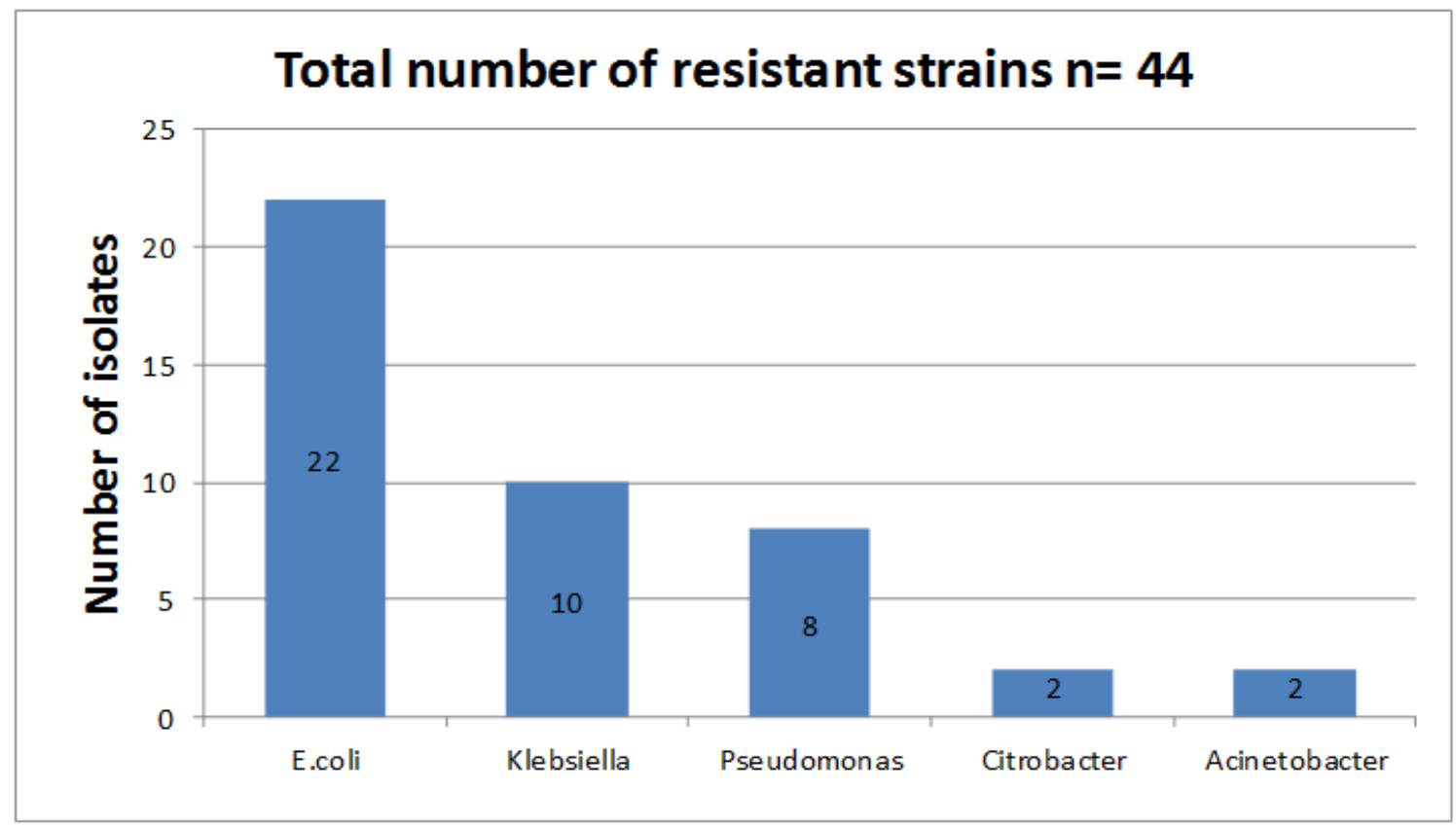

A comparison study between modified Hodge test and Carba NP test done by Bayramoğlu G et al., showed that the Carba NP test had a sensitivity of $93.9 \%$ to that of modified hodge test $90 \%$ concluding that the Carba NP test had a higher sensitivity, more reliant and non-time consuming as compared to modified hodge test. (Gülçin, 2016) concluded in this study that the Carba NP test detected all carbapenemase producers regardless of the type of the carbapenemase and of their mucoid phenotype (overall, there was $100 \%$ sensitivity and $100 \%$ specificity for carbapenemase producers, including OXA-48 producers, in a comparison with PCR results) by using $1.5 \mathrm{ml}$ eppendorf tubes instead of 96 well mictotitre plate. (Dortet et al., 2014) mentioned a sensitivity rate of $88 \%$ sensitivity he further mentioned that the sensitivity rate increases if the bacterial load from the isolate was more, in our study one standard loop size $(10 \mu \mathrm{g})$ was enough to confirm resistance. (Osterblad et al., 2014) confirmed a sensitivity of $100 \%$, similar in our study, when compared with modified hodgetest $98 \%$, specificity of Carba NP test showed a higher significance of $100 \%$ to that of modified hodge test $80 \%$, thus further strengthening the effectiveness of Carba NP test as a tool for detection (Vasoo et al., 2013).

The rapid Carba NP test is an easy screening method for carbapenemase producing bacteria and holds a higher sensitivity rate and cost effective than that of the phenotypic methods employed for detection of carbapenemase production.

This test can be implicated across for rapid screening and detection of carbapenemase producing bacteria and can help in early identification to prevent cross resistance to other patients by isolation of those resistant. Further evaluation of the Carba NP test can be done with PCR studies.

\section{Acknowledgement}

We thank SRM University for their constant support and for providing the fund necessary for completion of the project. 
We also thank the Hospital and Department of Microbiology for their support and cooperation.

\section{References}

Abdel Ghani, et al., Comparison of the Carba NP, Modified Carba NP, and Updated Rosco Neo-Rapid Carb Kit Tests for Carbapenemase Detection. Journal of Clinical Microbiology, 2015; 53(11), pp. 3539-3542.

Dortet, L., et al., "Further Proofs of Concept for the Carba NP Test". Antimicrobial Agents and Chemotherapy 2014; 58, 1269-1269.

Gülçin. "Comparison of the Modified Hodge Test and the Carba NP Test for Detection of Carbapenemases in Enterobacteriaceae Isolates". Mikrobiyoloji Bulteni 2016; 50, 1-10.

Kumarasamy, K., et al., Emergence of a new antibiotic resistance mechanism in India, Pakistan, and the UK: a molecular, biological, and epidemiological study. Lancet Infect Dis. 2010; Sep; 10, 597602.

Nair, P. Prevalence of carbapenem resistant Enterobacteriaceae from a tertiary care hospital in Mumbai, India. Journal of Microbiology and Infectious Diseases, 2013; 03(04), pp.207-210.

Nordmann Patrice et al., "Rapid Detection of Carbapenemase- Producing Enterobacteriaceae". Emerging Infectious Diseases 2012; 18, 1503-1507.

Nordmann, P., et al., Global Spread of Carbapenemase- producing Enterobacteriaceae. Emerging Infectious Diseases 2011; 17, 1791-1798.
Osterblad, M., et al., Evaluation of the Carba NP Test for Carbapenemase Detection. Antimicrobial Agents and Chemotherapy. 2014; 58, 7553-7556.

Porwal, R., et al., Carbapenem resistant Gramnegative bacteremia in an Indian intensive care unit: A review of the clinical profile and treatment outcome of 50 patients. Indian Journal of Critical Care Medicine, 2014; 18(11), p.820.

Shetty, A., "Evaluation of Carba NP Test for Rapid Detection of Carbapenemase Producing Enterobacteriaceae". Indian Journal of Medical Microbiology 2015; 33, 603.

Taneja, N., et al., Occurrence of blaNDM-1 \& absence of blaKPCgenes encoding carbapenem resistance in uropathogens from a tertiary care centre from north India. Indian Journal of Medical Research, 2015; 142, 336.

Tijet, N., "Evaluation of the Carba NP Test for Rapid Detection of CarbapenemaseProducing Enterobacteriaceae and Pseudomonas Aeruginosa". Antimicrobial Agents and Chemotherapy 2013; 57, 4578-4580

Vasoo, S., et al., Comparison of a Novel, Rapid Chromogenic Biochemical Assay, the Carba NP Test, with the Modified Hodge Test for Detection of CarbapenemaseProducing Gram-Negative Bacilli. Journal of Clinical Microbiology 2013; 51, 3097-3101

$\mathrm{Xu}$, Y., et al., Epidemiology of carbapenem resistant Enterobacteriaceae (CRE) during 2000-2012 in Asia. Journal of Thoracic Disease 2014; 7, 376-385.

\section{How to cite this article:}

Mangayarkarasi, V., Stephen Pradeep Moses, S.R. Swarna, K. Kalaiselvi and Shaweez Fathima, S. 2018. In-House Standardization of Carba NP Test for Carbapenemase Detection in Gram Negative Bacteria. Int.J.Curr.Microbiol.App.Sci. 7(01): 2876-2881. doi: https://doi.org/10.20546/ijcmas.2018.701.342 